\title{
Temporal dissipative solitons in a three-level atomic medium confined in a photonic-band-gap fiber
}

\author{
M. Facão* and S. Rodrigues ${ }^{\dagger}$ \\ Departamento de Física, Universidade de Aveiro and I3N, Campus Universitário de Santiago, 3810-193 Aveiro, Portugal
}

M. I. Carvalho

DEEC/FEUP and INESC TEC, Universidade do Porto, Rua Dr. Roberto Frias, 4200-465 Porto, Portugal

(Received 1 October 2014; published 20 January 2015)

\begin{abstract}
We obtained a propagation equation for an optical pulse at an electromagnetically induced transparency window guided on a gas-filled hollow-core photonic crystal fiber. This equation admits dissipative solitons whose analytical expression was also obtained. Depending on the parameter region, they may be stable or unstable. We simulated a typical experimental arrangement and found some cases for which the equation parameters are such that it admits stable solitons.
\end{abstract}

DOI: 10.1103/PhysRevA.91.013828

PACS number(s): 42.81.Dp, 42.50.Gy, 42.65.Hw

\section{INTRODUCTION}

Electromagnetically induced transparency (EIT) is an optical effect based on quantum interference that provides a narrow spectral transparent window within an absorption medium [1]. This phenomenon was first proposed in 1990 [2] and was observed in 1991 [3], and since then, it has attracted a lot of attention from the scientific community due to its unique properties, such as a sharp variation in dispersion and large nonlinear properties that make it extremely appealing for a large number of applications, including slow light [4], quantum memory [5], all-optical switching [6,7], and controlled light storage $[8,9]$.

The EIT effect can be considerably enhanced when the active medium is confined in a small region, such as a hollow planar waveguide $[10,11]$ or hollow-core photonic crystal fibers (HC-PCF) [12-14]. Moreover, further improvements are possible when Doppler broadening is avoided by filling the waveguide with cold atoms. However, the small dimensions of the region enclosing the active medium can make the adsorption of atoms to fiber walls significant, thus increasing dephasing rates. This process can be considerably reduced if the fiber walls are coated. Ghosh et al. managed to obtain EIT with nanowatt power levels by coating the fiber core with organosilane and, furthermore, by using a light-induced atomic desorption process that releases the atoms into the core [14]. Another scheme to avoid adsorption was recently proposed by Bajcsy et al. and uses a dipole trap along the fiber axis that attracts atoms away from the walls [7].

Besides reducing the optical intensities required to observe EIT, enclosing the active medium in a HC-PCF also offers the advantage of gaining better control over the dispersive properties. In effect, these can be manipulated to some degree by the modification of the fiber geometry, that is, the core and hole size, as well as the hole spacing, which can be extremely relevant in some applications that depend on the dispersive profile.

\footnotetext{
*mfacao@ua.pt

†silviamgr@ua.pt

${ }^{\ddagger}$ mines@fe.up.pt
}

Here, we address the EIT effect for a $\Lambda$ medium confined in a HC-PCF. By using a multiscale perturbation technique on both the density matrix time evolution and the probe field propagation equations, we obtain the equation for the slowly varying pulse envelope, which has the form of a complex Ginzburg-Landau equation (CGLE) with an additional term associated with the imaginary part of the group velocity. As expected, the parameters in this equation depend not only on the active medium and control field but also on the fiber characteristics. We then investigate analytical solutions of this equation, namely, their form, parameter region of existence, and stability. Using typical values for a three-level $\Lambda$ atomic system enclosed in a HC-PCF, we search for possible stable pulse solutions.

This article is structured as follows: Sec. II contains the derivation of the propagation equation for the probe field. In Sec. III we present the analytical solutions of the latter equation. In Sec. IV we show some simulation results for typical EIT arrangement on a HC-PCF, and in Sec. V we summarize the main results of our work.

\section{PROPAGATION MODEL}

Consider a three-level atomic system in the $\Lambda$ configuration as illustrated in Fig. 1 and two optical fields in close resonance with the transitions 1-3 and 2-3, namely, the probe $\mathcal{E}=\frac{1}{2}\left[\bar{E}(t, \mathbf{r}) e^{i\left(\beta_{p} z-\omega_{p} t\right)}+\right.$ c.c. $]$ and the control field $\mathcal{E}_{c}=\frac{1}{2}\left[\bar{E}_{c}(\mathbf{r}) e^{i\left(\beta_{c} z-\omega_{c} t\right)}+\right.$ c.c. $]$, respectively, where $\omega_{p, c}$ and $\beta_{p, c}$ are the angular frequencies and propagation constants. Actually, we consider a continuous-wave control field and a pulsed probe field, both guided inside a HC-PCF filled with a gas whose molecules have a $\Lambda$ configuration of energy levels. In the dipolar moment approximation, the Hamiltonian of the interaction is given by

$$
\begin{aligned}
\hat{H}_{\text {int }}= & -\frac{\hbar}{2}\left[\Omega_{p}(t, \mathbf{r}) e^{i\left(\omega_{31}-\omega_{p}\right) t+i \beta_{p} z}|3\rangle\langle 1|\right. \\
& \left.+\Omega_{c}(\mathbf{r}) e^{i\left(\omega_{32}-\omega_{c}\right) t+i \beta_{c} z}|3\rangle\langle 2|+\text { H.c. }\right],
\end{aligned}
$$

where $\Omega_{p, c}$ are the Rabi frequencies associated with the corresponding transition field and are given by $\Omega_{p}=\bar{E} \mu_{13} / \hbar$ and $\Omega_{c}=\bar{E}_{c} \mu_{23} / \hbar$, where $\mu_{13}$ and $\mu_{23}$ are the electric 


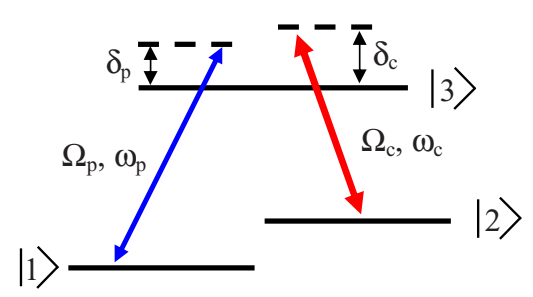

FIG. 1. (Color online) Energy-level diagram and excitation scheme of the three-level $\Lambda$ system considered.

dipole moments and $\omega_{31}$ and $\omega_{32}$ are the frequencies, which correspond to transitions 3-1 and 3-2, respectively.

The time evolution of the density operator in the interaction picture is given by

$$
\frac{d \hat{\rho}_{I}}{d t}=\frac{1}{i \hbar}\left[\hat{H}_{\mathrm{int}}, \hat{\rho}_{I}\right]+\hat{\mathcal{L}}\left(\hat{\rho}_{I}\right),
$$

where $\hat{\mathcal{L}}$ is the Lindblad superoperator that describes the relaxation and decoherence processes. The Lindblad term is given in the Appendix for the cases considered here, i.e., spontaneous emission from level 3 to levels 2 and 1 and energy-conserving dephasing effects. Hence, the evolution equations read

$$
\begin{gathered}
\frac{d \rho_{11}}{d t}=-i \frac{\Omega_{p}}{2} \tilde{\rho}_{13}+i \frac{\Omega_{p}^{*}}{2} \tilde{\rho}_{31}+\frac{\gamma_{33}}{2} \rho_{33}, \\
\frac{d \tilde{\rho}_{12}}{d t}=-i \frac{\Omega_{c}}{2} \tilde{\rho}_{13}+i \frac{\Omega_{p}^{*}}{2} \tilde{\rho}_{32}-\Gamma_{12} \tilde{\rho}_{12}, \\
\frac{d \tilde{\rho}_{13}}{d t}=-i \frac{\Omega_{p}^{*}}{2} \rho_{11}-i \frac{\Omega_{c}^{*}}{2} \tilde{\rho}_{12}+i \frac{\Omega_{p}^{*}}{2} \rho_{33}-\Gamma_{13} \tilde{\rho}_{13}, \\
\frac{d \rho_{22}}{d t}=-i \frac{\Omega_{c}}{2} \tilde{\rho}_{23}+i \frac{\Omega_{c}^{*}}{2} \tilde{\rho}_{32}+\frac{\gamma_{33}}{2} \rho_{33}, \\
\frac{d \tilde{\rho}_{23}}{d t}=-i \frac{\Omega_{p}^{*}}{2} \tilde{\rho}_{21}-i \frac{\Omega_{c}^{*}}{2} \rho_{22}+i \frac{\Omega_{c}^{*}}{2} \rho_{33}-\Gamma_{23} \tilde{\rho}_{23}, \\
\frac{d \rho_{33}}{d t}=i \frac{\Omega_{p}}{2} \tilde{\rho}_{13}+i \frac{\Omega_{c}}{2} \tilde{\rho}_{23}-i \frac{\Omega_{p}^{*}}{2} \tilde{\rho}_{31}-i \frac{\Omega_{c}^{*}}{2} \tilde{\rho}_{32}-\gamma_{33} \rho_{33},
\end{gathered}
$$

where $\Gamma_{12}=\gamma_{12}-i \Delta, \Gamma_{13}=\gamma_{13}+i \delta_{p}, \Gamma_{23}=\gamma_{23}+i \delta_{c}$ are the complex decoherence parameters, with $\gamma_{i j}$ being the total decay rates, considering $S_{i j}$ and $D_{i j}$ as given in the Appendix, and $\delta_{p}=\omega_{p}-\omega_{31}, \delta_{c}=\omega_{c}-\omega_{32}$, and $\Delta=\delta_{c}-\delta_{p}$ are the detunings between the optical fields and level-transition frequencies. In writing the population evolution equations we assumed equal decay rates from the highest energy level to the lower ones. The tilde versions of the density matrix coefficients correspond to the initial ones by factoring out the rapid oscillations, namely,

$$
\begin{aligned}
& \tilde{\rho}_{12}=\rho_{12} e^{i \Delta t-i \beta_{c} z+i \beta_{p} z}, \quad \tilde{\rho}_{13}=\rho_{13} e^{-i \delta_{p} t+i \beta_{p} z}, \\
& \tilde{\rho}_{23}=\rho_{23} e^{-i \delta_{c} t+i \beta_{c} z} .
\end{aligned}
$$

For convenience, we shall write the above system of equations in the following matrix representation:

$$
\frac{d \rho}{d t}=\left(A_{0}+A_{p}\right) \rho,
$$

where $\rho$ stands for the vector of the density matrix coefficients $\rho_{i i}$ and $\tilde{\rho}_{i j}$ and $A_{0}$ and $A_{p}$ are the $\Omega_{p}$-independent and -dependent parts of the system matrix, respectively.

In what follows we will consider the evolution of the complex envelope, and we will denote it by $\mathcal{E}$, that is, $\mathcal{E}=\bar{E}(t, \mathbf{r}) e^{i\left(\beta_{p} z-\omega_{p} t\right)}$, where $\bar{E}(t, \mathbf{r})$ is the slowly varying part of that complex envelope. $\mathcal{E}$ will propagate according to

$$
\frac{\partial^{2} \mathcal{E}}{\partial z^{2}}+\nabla_{t}^{2} \mathcal{E}-\frac{1}{c^{2}} \frac{\partial^{2} \mathcal{E}}{\partial t^{2}}=\mu_{0} \frac{\partial^{2} P}{\partial t^{2}},
$$

where $\nabla_{t}$ is the transverse Laplacian, $c$ is the vacuum light velocity, $\mu_{0}$ is the magnetic permeability, and the polarization is $P=\epsilon_{0} \chi_{\text {solid }} \mathcal{E}$ in the solid regions of the fiber and $P=$ $2 N \mu_{13} \rho_{31}^{S}$ in the gas regions. Here, $N$ is the density of gas molecules, and $\rho_{31}^{S}$ is the corresponding density matrix coefficient in the Schrödinger representation. Note that $\hat{\rho}^{I}$ in the interaction picture is related to $\hat{\rho}^{S}$ in the Schrödinger picture by $\hat{\rho}^{I}=e^{i \hat{H}_{A} t / \hbar} \hat{\rho}^{S} e^{-i \hat{H}_{A} t / \hbar}$, where $\hat{H}_{A}$ is the atomic Hamiltonian. Hence, $\rho_{31}^{S}=\rho_{31} e^{-i \omega_{31} t}=\tilde{\rho}_{31} e^{i\left(\beta_{p} z-\omega_{p} t\right)}$, where $\tilde{\rho}_{31}=\tilde{\rho}_{13}^{*}$.

Furthermore, Eqs. (2) and (3) will be solved using a multiscale perturbation method identical to the one used in [15] with the new variables $t_{0}=t, t_{1}=\delta t, t_{2}=\delta^{2} t, z_{0}=z, z_{1}=$ $\delta z, z_{2}=\delta^{2} z$, where $\delta$ is a dimensionless small parameter, and a power series expansion for $\bar{E}$ and $\rho$ of the form

$$
\begin{gathered}
\bar{E}\left(t_{1}, t_{2}, x, y, z_{1}, z_{2}\right)=\delta \bar{E}^{(1)}+\delta^{2} \bar{E}^{(2)}+\delta^{3} \bar{E}^{(3)}+\cdots, \\
\boldsymbol{\rho}\left(t_{1}, t_{2}, x, y, z_{1}, z_{2}\right)=\boldsymbol{\rho}^{(0)}+\delta \boldsymbol{\rho}^{(1)}+\delta^{2} \boldsymbol{\rho}^{(2)}+\delta^{3} \boldsymbol{\rho}^{(3)}+\cdots,
\end{gathered}
$$

where $\rho$ is the vector of the density matrix coefficients considered above, obtained by factoring out the rapid oscillatory term. Note that this term oscillates in the scales of $t_{0}$ and $z_{0}$. This implies that $\mathcal{E}$ is also given by a similar power series expansion and that, in contrast to the slowly varying $\bar{E}$ and $\rho, \mathcal{E}$ also depends on $t_{0}$ and $z_{0}$ through the rapid oscillatory term. In order zero, we have only the equation for $\rho^{(0)}$ that reads

$$
A_{0} \rho^{(0)}=0
$$

and gives

$$
\rho_{11}^{(0)}=1, \quad \rho_{i j}^{(0)}=0 \quad \text { for } i, j \neq 1 .
$$

In first order in $\delta$, the equation for $\rho^{(1)}$ reads

$$
A_{0} \boldsymbol{\rho}^{(1)}=-A_{p}\left(\bar{E}^{(1)}\right) \boldsymbol{\rho}^{(0)} .
$$

The solution of the above system is $\tilde{\rho}_{31}^{(1)}=\left(L \mu_{13} / \hbar\right) \bar{E}^{(1)}$, which in the Schrödinger picture is $\rho_{31}^{S(1)}=\left(L \mu_{13} / \hbar\right) \mathcal{E}^{(1)}$, where $L$ is given by

$$
L=\frac{-2\left(\Delta-i \gamma_{12}\right)}{4\left(\Delta-i \gamma_{12}\right)\left(\delta_{p}+i \gamma_{13}\right)+\left|\Omega_{c}\right|^{2}} .
$$

In first order in $\delta$, the equation for the optical field is

$$
\frac{\partial^{2} \mathcal{E}^{(1)}}{\partial z_{0}^{2}}+\nabla_{t}^{2} \mathcal{E}^{(1)}-\frac{1}{c^{2}} \frac{\partial^{2} \mathcal{E}^{(1)}}{\partial t_{0}^{2}}=\mu_{0} \epsilon_{0} \chi \frac{\partial^{2} \mathcal{E}^{(1)}}{\partial t_{0}^{2}},
$$

where $\chi \equiv \chi\left(\omega_{p} ; x, y\right)$ is equal to the electric susceptibility of the solid material or equal to $2 c^{2} \mu_{0} N \mu_{13}^{2} L / \hbar$ if $(x, y)$ is in the solid or gas region, respectively. The above equation has 
solutions of the form $\mathcal{E}^{(1)}=F(x, y) E^{(1)}\left(t_{1}, t_{2}, z_{1}, z_{2}\right) e^{-i \omega_{p} t+i \beta_{p} z}$ if

$$
-\beta_{p}^{2} F E^{(1)}+E^{(1)} \nabla_{t}^{2} F+\frac{\omega_{p}^{2}}{c^{2}} n^{2}\left(\omega_{p} ; x, y\right) F E^{(1)}=0,
$$

where

$$
n^{2}\left(\omega_{p} ; x, y\right)=1+\chi\left(\omega_{p} ; x, y\right) .
$$

Dividing Eq. (4) by $E^{(1)}$, we obtain an eigenvalue equation for $F$, whose eigenvalue is the propagation constant $\beta_{p}$. This equation may be solved for the adequate fiber geometry, and the dispersion relation $\beta_{p}(\omega)$ may be retrieved. Furthermore, multiplying by $F^{*}$ and integrating in $x$ and $y$ give

$$
\beta_{p}^{2}=\frac{\int F^{*} \nabla_{t}^{2} F d x d y+k_{p}^{2} \int n^{2}|F|^{2} d x d y}{\int|F|^{2} d x d y},
$$

where $k_{p}=\omega_{p} / c$. In second order in $\delta$ we obtain

$$
A_{0} \boldsymbol{\rho}^{(2)}=\frac{\partial \boldsymbol{\rho}^{(1)}}{\partial t_{1}}-A_{p}\left(\bar{E}^{(1)}\right) \boldsymbol{\rho}^{(1)}-A_{p}\left(\bar{E}^{(2)}\right) \boldsymbol{\rho}^{(0)}
$$

whose solution gives

$$
\tilde{\rho}_{31}^{(2)}=\frac{\mu_{13}}{\hbar}\left(L \bar{E}^{(2)}+B \frac{\partial \bar{E}^{(1)}}{\partial t_{1}}\right)
$$

where the expression for $B$ is shown in the Appendix. For the probe field, we have

$$
\begin{aligned}
& \frac{\partial \mathcal{E}^{(2)}}{\partial z_{0}^{2}}+\nabla_{t}^{2} \mathcal{E}^{(2)}-\frac{1}{c^{2}}(1+\chi) \frac{\partial^{2} \mathcal{E}^{(2)}}{\partial t_{0}^{2}} \\
& =-2 \frac{\partial^{2} \mathcal{E}^{(1)}}{\partial z_{0} \partial z_{1}}+\frac{2}{c^{2}} \frac{\partial^{2} \mathcal{E}^{(1)}}{\partial t_{0} \partial t_{1}}+\frac{i}{c^{2}} \frac{d \chi}{d \omega_{p}} \frac{\partial^{3} \mathcal{E}^{(1)}}{\partial t_{0}^{2} \partial t_{1}}+\frac{2 \chi}{c^{2}} \frac{\partial^{2} \mathcal{E}^{(1)}}{\partial t_{0} \partial t_{1}}
\end{aligned}
$$

In order to avoid secular terms for $\mathcal{E}^{(2)}$, the right-hand side of the equation should be equal to zero, which gives

$$
-2 i \beta_{p} F \frac{\partial E^{(1)}}{\partial z_{1}}-\frac{2 i \omega_{p}}{c^{2}}\left(1+\chi+\frac{\omega_{p}}{2} \frac{d \chi}{d \omega_{p}}\right) F \frac{\partial E^{(1)}}{\partial t_{1}}=0 .
$$

Multiplying by $F^{*}$ and integrating in $x$ and $y$, we obtain

$$
i \frac{\partial E^{(1)}}{\partial z_{1}}+i \beta_{p}^{\prime} \frac{\partial E^{(1)}}{\partial t_{1}}=0
$$

where $\beta_{p}^{\prime}=\partial \beta_{p} / \partial \omega_{p}$. Without any loss of generality, we assume $\mathcal{E}^{(2)}=0$ and obtain the following equation in third order

$$
A_{0} \boldsymbol{\rho}^{(3)}=\frac{\partial \boldsymbol{\rho}^{(2)}}{\partial t_{1}}+\frac{\partial \boldsymbol{\rho}^{(1)}}{\partial t_{2}}-A_{p}\left(\bar{E}^{(1)}\right) \boldsymbol{\rho}^{(2)}-A_{p}\left(\bar{E}^{(3)}\right) \boldsymbol{\rho}^{(0)},
$$

whose solution gives

$$
\begin{aligned}
\tilde{\rho}_{31}^{(3)}= & \frac{\mu_{13}}{\hbar}\left(L \bar{E}^{(3)}+\frac{\mu_{13}^{2}}{\hbar^{2}} G\left|\bar{E}^{(1)}\right|^{2} \bar{E}^{(1)}\right. \\
& \left.+B \frac{\partial \bar{E}^{(1)}}{\partial t_{2}}+C \frac{\partial^{2} \bar{E}^{(1)}}{\partial t_{1}^{2}}\right)
\end{aligned}
$$

where the expressions for $G$ and $C$ can be found in the Appendix. Also in third order in $\delta$, the probe field equation is

$$
\begin{aligned}
\frac{\partial \mathcal{E}^{(3)}}{\partial z_{0}^{2}} & +\nabla_{t}^{2} \mathcal{E}^{(3)}-\frac{1}{c^{2}}(1+\chi) \frac{\partial^{2} \mathcal{E}^{(3)}}{\partial t_{0}^{2}} \\
= & {\left[-2 i \beta_{p} F \frac{\partial E^{(1)}}{\partial z_{2}}-F \frac{\partial^{2} E^{(1)}}{\partial z_{1}^{2}}\right.} \\
& -\frac{2 i \omega_{p}}{c^{2}}\left(1+\chi+\frac{\omega_{p}}{2} \frac{d \chi}{d \omega_{p}}\right) F \frac{\partial E^{(1)}}{\partial t_{2}} \\
& +\frac{1}{c^{2}}\left(1+\chi+2 \omega_{p} \frac{d \chi}{d \omega_{p}}+\frac{\omega_{p}^{2}}{2} \frac{d^{2} \chi}{d \omega_{p}^{2}}\right) F \frac{\partial^{2} E^{(1)}}{\partial t_{1}^{2}} \\
& \left.-\mathcal{G}|F|^{2} F\left|E^{(1)}\right|^{2} E^{(1)}\right] e^{i\left(\beta_{p} z-\omega_{p} t\right)},
\end{aligned}
$$

where $\mathcal{G}=2 \omega_{p}^{2} \mu_{0} N \mu_{13}^{4} G / \hbar^{3}$. Since we expect that the light is well confined inside the core, we will not consider the nonlinear Kerr effect in the cladding. For the same reasoning as above, namely, to avoid secular terms for $\mathcal{E}^{(3)}$, the righthand side should be zero. Moreover, multiplying by $F^{*}$ and integrating in $x$ and $y$ yield

$$
\begin{aligned}
& i \frac{\partial E^{(1)}}{\partial z_{2}}+\frac{1}{2 \beta_{p}} \frac{\partial^{2} E^{(1)}}{\partial z_{1}^{2}}+i \beta_{p}^{\prime} \frac{\partial E^{(1)}}{\partial t_{2}}-\left(\frac{1}{2} \beta_{p}^{\prime \prime}+\frac{\left(\beta_{p}^{\prime}\right)^{2}}{2 \beta_{p}}\right) \\
& \times \frac{\partial^{2} E^{(1)}}{\partial t_{1}^{2}}+g\left|E^{(1)}\right|^{2} E^{(1)}=0
\end{aligned}
$$

where

$$
g=\frac{\int \mathcal{G}|F|^{4} d x d y}{2 \beta_{p} \int|F|^{2} d x d y}
$$

and $\beta_{p}^{\prime \prime}=\partial^{2} \beta_{p} / \partial \omega_{p}^{2}$. We first note that the term of the second derivative with respect to $z_{1}$ and the one containing $\left(\beta_{p}^{\prime}\right)^{2}$ may cancel due to (6). Then adding Eq. (8) multiplied by $\delta^{2}$ and Eq. (10) multiplied by $\delta^{3}$, we obtain

$$
i \frac{\partial E}{\partial z}+i \beta_{p}^{\prime} \frac{\partial E}{\partial t}-\frac{1}{2} \beta_{p}^{\prime \prime} \frac{\partial^{2} E}{\partial t}+g|E|^{2} E=0 .
$$

At this stage we should note that since the linear response $L$ of the gas is complex, our propagation constant should also be a complex quantity. Let us write it as $\beta_{p}=\beta_{r}+i \beta_{i}$, with $\beta_{r}$ and $\beta_{i}$ being real. The imaginary part will lead to pulse attenuation proportional to $\exp \left(-\beta_{i} z\right)$, but since $e^{i\left(\beta_{p} z-\omega_{p} t\right)}$ was factored out, the solution of the above equation is not attenuated. Here, we reintroduce $\exp \left(-i \beta_{i} z\right)$ in the equation by defining a new envelope $E^{\prime}=E \exp \left(-i \beta_{i} z\right)$. Moreover, the pulse will propagate with a group velocity equal to $1 / \beta_{r}^{\prime}$. Hence, making the change of variables $\tau=t-\beta_{r}^{\prime} z$, the equation for $E^{\prime}$ is

$$
i \frac{\partial E^{\prime}}{\partial z}-\frac{1}{2} \beta_{p}^{\prime \prime} \frac{\partial^{2} E^{\prime}}{\partial \tau^{2}}+g\left|E^{\prime}\right|^{2} E^{\prime}=-i \beta_{i} E^{\prime}+\beta_{i}^{\prime} \frac{\partial E^{\prime}}{\partial \tau} .
$$

Applying the usual change of variables, i.e., $U=$ $\sqrt{\epsilon_{0} c \bar{n} \int|F|^{2} d x d y / 2} E^{\prime}$, where $\bar{n}$ is an average real refractive index in the illuminated region, and separating the real and 
imaginary coefficients, we obtain

$$
\begin{aligned}
& i \frac{\partial U}{\partial z}-\frac{1}{2} \beta_{r}^{\prime \prime} \frac{\partial^{2} U}{\partial \tau^{2}}-\frac{i}{2} \beta_{i}^{\prime \prime} \frac{\partial^{2} U}{\partial \tau^{2}}+\gamma_{r}|U|^{2} U+i \gamma_{i}|U|^{2} U \\
& \quad=-i \beta_{i} U+\beta_{i}^{\prime} \frac{\partial U}{\partial \tau}
\end{aligned}
$$

with $\gamma_{r}+i \gamma_{i}=2 g /\left(\epsilon_{0} c \bar{n} \int|F|^{2} d x d y\right)$. Also, applying

$$
q=\tau_{0} \sqrt{\left|\frac{\gamma_{r}}{\beta_{r}^{\prime \prime}}\right|} U, \quad Z=\frac{z}{z_{0}}, \quad T=\frac{\tau}{\tau_{0}},
$$

and

$$
z_{0}=\frac{\tau_{0}^{2}}{\left|\beta_{r}^{\prime \prime}\right|}
$$

we arrive at the dimensionless form for the propagation equation that takes the form of the well-known cubic GinzburgLandau equation with an imaginary group-velocity term:

$$
\begin{aligned}
& i \frac{\partial q}{\partial Z}-\frac{D}{2} \frac{\partial^{2} q}{\partial T^{2}}+s|q|^{2} q \\
& \quad=i \alpha q+\xi \frac{\partial q}{\partial T}+i \nu \frac{\partial^{2} q}{\partial T^{2}}+i \sigma|q|^{2} q .
\end{aligned}
$$

It should be pointed out that, with the exception of an extra term associated with diffraction, this equation is similar to the one obtained by Huang et al. when considering a third-order approximation [15]. Here, $D=\beta_{r}^{\prime \prime} /\left|\beta_{r}^{\prime \prime}\right|$ is 1 for normal group-velocity dispersion $\left(\beta_{r}^{\prime \prime}>0\right)$ and -1 for anomalous group-velocity dispersion $\left(\beta_{r}^{\prime \prime}<0\right) ; s=\gamma_{r} /\left|\gamma_{r}\right|$ is also $\pm 1, \alpha=-\beta_{i} z_{0}, \xi=z_{0} \beta_{i}^{\prime} / \tau_{0}, v=\beta_{i}^{\prime \prime} /\left(2\left|\beta_{r}^{\prime \prime}\right|\right)$, and $\sigma=-\gamma_{i} /\left|\gamma_{r}\right|$. Note that the parameters in this equation depend not only on the three-level gas and control field value but also on the fiber geometry, which suggests that the dispersive and nonlinear properties of a HC-PCF filled with a $\Lambda$ system can be manipulated to a significant extent. In the following we will analyze the solutions of Eq. (12).

\section{SOLITON SOLUTIONS}

As long as $D$ and $s$ have symmetrical signs, i.e., $D s=-1$, the left-hand side of Eq. (12) is the nonlinear Schrödinger equation (NLSE) that admits bright solitons. If $\xi=0$, the equation is the cubic CGLE, which admits pulselike solutions for both $D s=-1$ and $D s=1$ [16]. In fact, its stationary solutions were written in terms of hyperbolic-shape pulses with a chirp parameter [16-18]. However, they are stable only when $D s=-1, \alpha=0$, and $v>0$, with this last condition required to assure stability in the frequency domain [18]. Furthermore, Eq. (12) also exhibits stationary solutions for finite $\xi$ and $\alpha=0$ in the form of a chirped traveling solution [19].

In our case, we cannot have $\alpha=0$ unless $\gamma_{12}=0$ and we are in the exact resonance, $\Delta=0$. But in this latter case, the nonlinear term would also be zero. Hence, we follow the search for stable solutions in the case $\alpha \neq 0$. First, we start by considering that $D s=-1$ and that the terms on the right-hand side of Eq. (12) are small enough to allow a perturbation approach around the hyperbolic secant soliton of the NLSE. Let us also assume the case $D=-1$ and $s=1$ and introduce the solution

$$
\begin{aligned}
q(T, Z)= & \eta(Z) \operatorname{sech}\left\{\eta(Z)\left[T-T_{0}(Z)\right]\right\} \\
& \times \exp [-i k(Z) T+i \zeta(Z)],
\end{aligned}
$$

and let us find the $Z$ evolution for $\eta(Z), k(Z), T_{0}(Z)$, and $\zeta(Z)$ using the procedure described in $[20,21]$. The results are

$$
\begin{gathered}
\frac{d \eta}{d Z}=2 \eta \alpha+\frac{4}{3} \sigma \eta^{3}-\frac{2}{3} \nu \eta\left(\eta^{2}+3 k^{2}\right)-2 k \eta \xi, \\
\frac{d k}{d Z}=-\frac{4}{3} v \eta^{2} k-\frac{2}{3} \eta^{2} \xi, \\
\frac{d T_{0}}{d Z}=-k, \quad \frac{d \zeta}{d Z}=\frac{1}{2}\left(\eta^{2}-k^{2}\right)+T_{0} \frac{d k}{d t} .
\end{gathered}
$$

The equations for $\eta$ and $k$ have two points of equilibrium, $\eta_{e}=0$ and any $k$ and

$$
\eta_{e}=\sqrt{\frac{3\left(\xi^{2}+4 \alpha \nu\right)}{4 v(\nu-2 \sigma)}}, \quad k_{e}=-\frac{\xi}{2 \beta} .
$$

Assuming $v>0$, there are real solutions for $\eta_{e}$ if $\xi^{2}+$ $4 \alpha \nu>0$ and $\nu-2 \sigma>0$ or if $\xi^{2}+4 \alpha \nu<0$ and $\nu-2 \sigma<0$. However, for this equilibrium point to be stable, it is necessary that $\xi^{2}+4 \alpha v>0$.

Then, motivated by the analytical results of [17-19], we look for solutions of Eq. (12) with a nonnegligible right-hand side and with both nonzero $\xi$ and nonzero $\alpha$ in the form

$$
q(T, Z)=A \operatorname{sech}^{1+i d}\left[B\left(T-\frac{Z}{v}\right)\right] \exp [i(K Z-\Omega T)],
$$

where $A, B, v, K, \Omega$, and the chirp parameter $d$ are all real. Substituting this expression in Eq. (12) and considering separately the equations for the real and imaginary parts, we obtain

$$
\begin{gathered}
\Omega=-\frac{\xi}{2 v}, \\
\frac{1}{v}=-\frac{D \xi}{2 v}, \\
B^{2}=\frac{\alpha+\frac{\xi^{2}}{4 v}}{v d^{2}-v-D d}, \\
B^{2}\left[\frac{D\left(d^{2}-1\right)}{2}+2 v d\right]+\frac{D \Omega^{2}}{2}, \\
A^{2}=\frac{3 B^{2} d\left(1+4 v^{2}\right)}{2(D \sigma+2 v s)},
\end{gathered}
$$

where $d$ satisfies

$$
(D \sigma+2 s v) d^{2}+3(2 \sigma v-s D) d-2(D \sigma+2 s v)=0 .
$$

Let us first consider the solutions corresponding to $D \sigma+$ $2 v s=0$. In this case, the chirp parameter is $d=0$, and we have $\sigma / \nu=-2 s / D= \pm 2$. From (14) we can conclude that $A^{2}=$ $2(\nu / \sigma) B^{2}$, implying that a chirp-free solution exists only when $s / D=-1$ and that it satisfies $A^{2}=B^{2}$. On the other hand, from (13), with $d=0$, we also have $B^{2}=-\left[\alpha+\xi^{2} /(4 v)\right] / \nu$, 


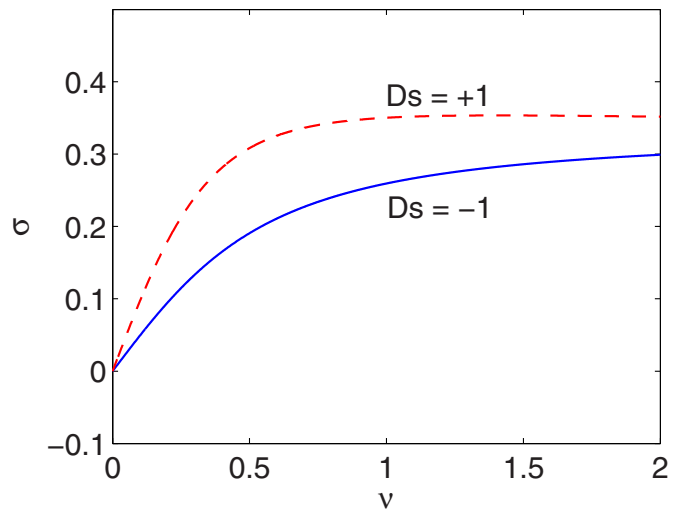

FIG. 2. (Color online) Curve of $\sigma_{s}$ defined by Eq. (19) when $D s=-1$ (blue solid line) and $D s=+1$ (red dashed line). The existence of solutions requires that $\sigma$ is above $\sigma_{s}$ when $\alpha+\frac{\xi^{2}}{4 v}<0$ or below $\sigma_{s}$ if $\alpha+\frac{\xi^{2}}{4 v}>0$.

which in turn requires $\alpha / v<-[\xi /(2 v)]^{2}$, that is, $\alpha / v<-\Omega^{2}$, for this type of solution to exist.

Similar constrains exist when $D \sigma+2 \nu s \neq 0$. In fact, since $A$ and $B$ are real, we must have

$$
\frac{d}{D \sigma+2 v s}>0
$$

and

$$
\frac{\alpha+\frac{\xi^{2}}{4 v}}{v d^{2}-v-D d}>0
$$

It is possible to show that the only solution of (15) satisfying condition (16) is given by

$$
d=\frac{3(s D-2 \sigma \nu)+\sqrt{9(s D-2 \sigma \nu)^{2}+8(D \sigma+2 s \nu)^{2}}}{2(D \sigma+2 \nu s)} .
$$

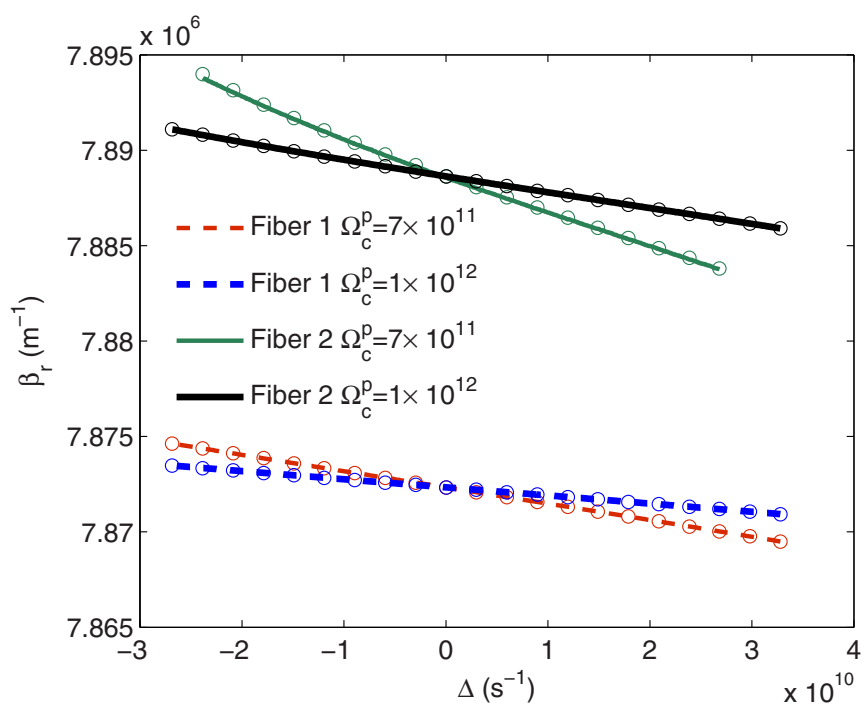

(a)
Equation (17) is satisfied if $\alpha+\frac{\xi^{2}}{4 v}>0$ and $v d^{2}-v-D d>$ 0 or if $\alpha+\frac{\xi^{2}}{4 v}<0$ and $v d^{2}-v-D d<0$. Even though the denominator of (17) has two roots, namely, $d^{-}=\frac{D-\sqrt{1+4 v^{2}}}{2 v}$ and $d^{+}=\frac{D+\sqrt{1+4 v^{2}}}{2 v}$, condition (16) implies that for a combination of parameters $(D, s, \nu, \sigma)$ only one of these two roots is allowed. In fact, again assuming that $v>0$ for stability reasons, the denominator will be positive when $D \sigma+2 s v>0$ for $d>d^{+}$and when $D \sigma+2 s v<0$ for $d<d^{-}$. Using (18) in each of these expressions reveals that if $\sigma$ is below the curve defined by

$$
\sigma_{s}=\frac{v\left(3 \sqrt{1+4 v^{2}}+D s\right)}{4+18 v^{2}}
$$

the denominator will be positive, while it will be negative above that curve. The curves defined by this equation for $D s=$ \pm 1 are represented in Fig. 2. A similar result was obtained in Ref. [16] for $s=+1$. Hence, for solutions to exist, $\sigma<\sigma_{s}$ and $\alpha+\frac{\xi^{2}}{4 v}>0$ or $\sigma>\sigma_{s}$ and $\alpha+\frac{\xi^{2}}{4 v}<0$.

Note that for small $v$ the curve $\sigma_{s}$ becomes $\sigma_{s}=\frac{v(3+D s)}{4}$, which for $D s=-1$ coincides with the boundary of stability $\sigma=\frac{v}{2}$ that was obtained using the perturbation analysis. We have performed a preliminary stability analysis that included the determination of the continuous spectrum of the spectral stability equations and direct numerical simulation of the evolution equation (12) using pseudospectral methods. This analysis showed that the solutions are stable for $\sigma<\sigma_{s}$ and $\alpha+\xi^{2} /(4 v)>0$; however, the continuous spectrum is stable for $v>0$ only if $\alpha+\xi^{2} /(4 v)<0$. Hence, in analogy to what has been proved for the cubic CGLE [16,18], we have a region of stable solitons and unstable linear waves and unstable solitons and stable linear waves. Fortunately, as was observed in simulations of Eq. (12), the instability of the linear waves is not very pronounced in the case of small $\alpha+\xi^{2} /(4 v)$.

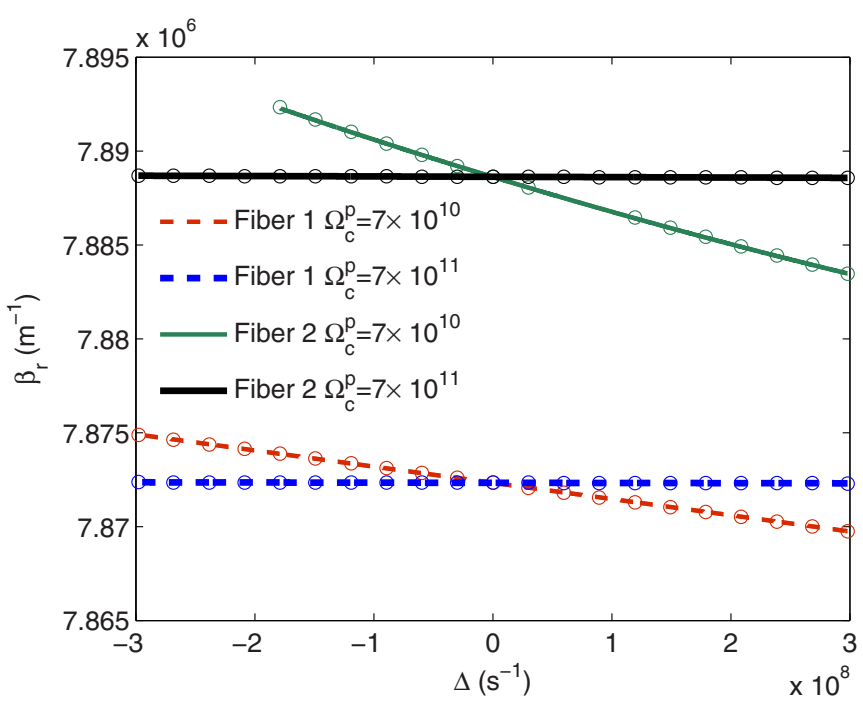

(b)

FIG. 3. (Color online) The real part of the propagation constant for both fibers and two peak values of the control Rabi frequency (in $\mathrm{s}^{-1}$ ). The population decay and dephasing rates are (a) all equal to $6.28 \times 10^{7} \mathrm{~s}^{-1}$ (case 1) and (b) $\gamma_{13}=\gamma_{23}=18.1 \times 10^{6} \mathrm{~s}^{-1}, \gamma_{33}=36.1 \times 10^{6} \mathrm{~s}^{-1}$ (case 2). Circles correspond to values determined numerically, and the lines correspond to fourth-degree polynomial fittings. 


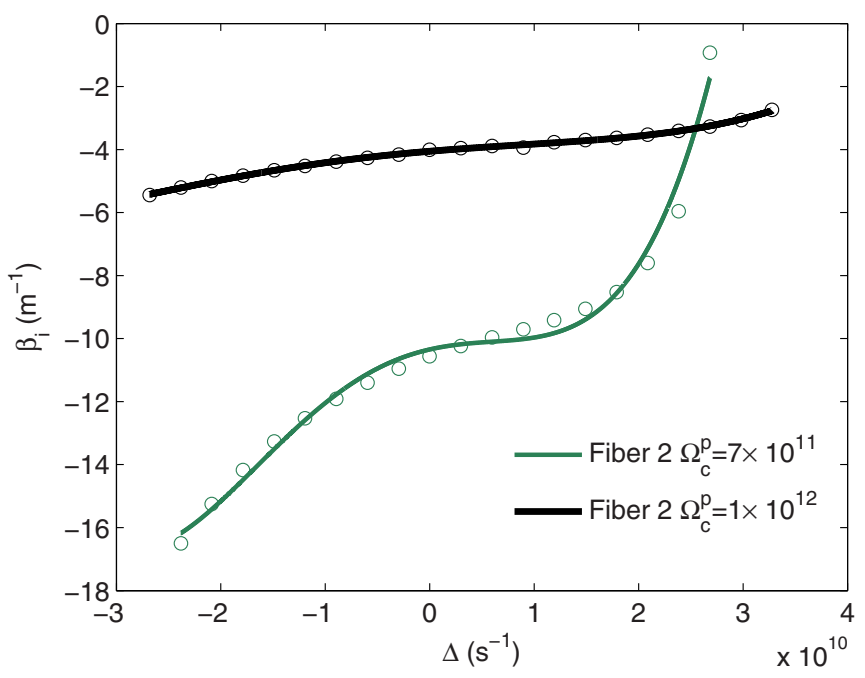

(a)

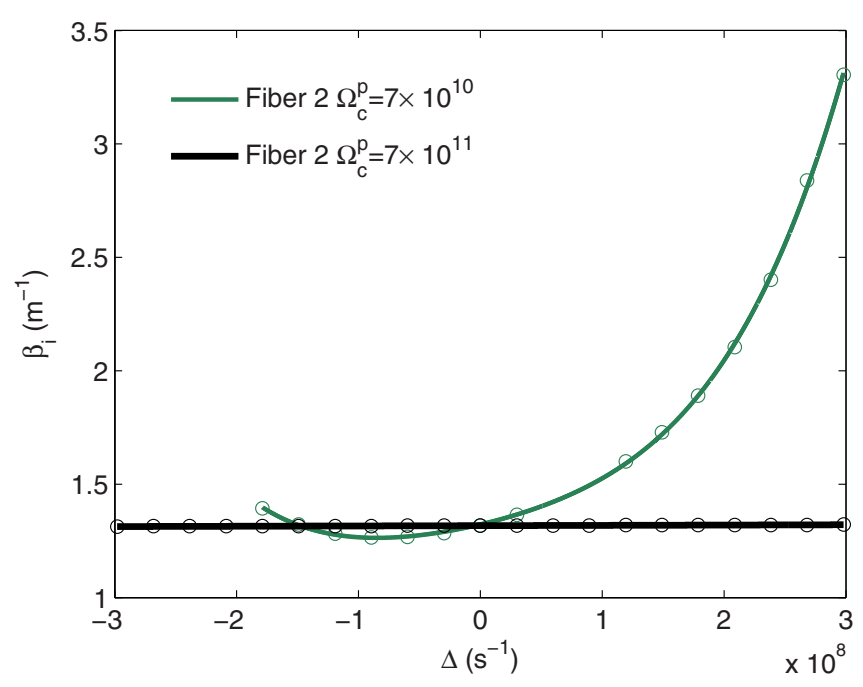

(b)

FIG. 4. (Color online) The imaginary part of the propagation constant for fiber 2 and two peak values of the control Rabi frequency (in $\mathrm{s}^{-1}$ ). The population decay and dephasing rates are (a) all equal to $6.28 \times 10^{7} \mathrm{~s}^{-1}$ (case 1) and (b) $\gamma_{13}=\gamma_{23}=18.1 \times 10^{6} \mathrm{~s}^{-1}, \gamma_{33}=$ $36.1 \times 10^{6} \mathrm{~s}^{-1}$ (case 2). Circles correspond to values determined numerically, and the lines correspond to fourth-degree polynomial fittings.

\section{RESULTS AND DISCUSSION}

In the following, we consider a gas of rubidium and its energy levels ${ }^{5} S_{1 / 2} F=2,{ }^{5} S_{1 / 2} F=3$, and ${ }^{5} P_{1 / 2}$ as $|1\rangle,|2\rangle$, and $|3\rangle$, respectively. For these $\Lambda$ levels, the control and probe wavelengths may be considered to be numerically identical and equal to $795 \mathrm{~nm}$, and $\mu_{13}=2.54 \times 10^{-29} \mathrm{C} \mathrm{m}$. We have also considered two photonic-band-gap fibers with hexagonal structured claddings whose solid material is silica. The pitches and hole diameters are 2.5 and 2.3 for fiber 1 and 4.0 and $3.9 \mu \mathrm{m}$ for fiber 2 , respectively. In both fibers, the core diameter is taken to be three times the hole diameter. The results below were all obtained by considering a fiber core filled with rubidium gas at 0.1 mbar of pressure, which in STP conditions gives $N=2.46 \times 10^{21} \mathrm{~m}^{-3}$. The cladding holes were considered to be filled with air. We also consider that the control field is in exact resonance with the energy difference between levels 2 and 3, i.e., $\delta_{c}=0$. Moreover, we set all $\gamma_{i j}$ equal to $6.28 \times 10^{7} \mathrm{~s}^{-1}$ (case 1 ), which is a typical value for room-temperature EIT experiments [14]. For comparison purposes, we also add the results obtained considering all $D_{i j}=0$ and $S_{31}=S_{32}=18.1 \times 10^{6} \mathrm{~s}^{-1}$ [22], such that $\gamma_{12}=$ $0, \gamma_{13}=\gamma_{23}=18.1 \times 10^{6} \mathrm{~s}^{-1}$, and $\gamma_{33}=36.1 \times 10^{6} \mathrm{~s}^{-1}$ (case 2).

We have determined the optical modes around the resonant wavelength for the two fibers using the refractive index (5) for several values of the control Rabi frequency and in both conditions of population decay and dephasing rates, using COMSOL MULTIPHYSICS [23]. The control field fundamental mode was obtained by considering the HC-PCF filled with air. Figures 3 and 4 show the real and imaginary parts of the propagation constant for some of these results. Our results show that $\beta_{r}$ closely follows the $\Delta$ dependency of the real part of $n$ given by Eq. (5), but the imaginary part $\beta_{i}$ is much more dependent on the control Rabi frequency and the fiber geometric parameters. Let us note that a positive imaginary part for $\beta$ or $n$ is loss and a negative one is gain. The EIT arrangement always gives a positive imaginary part of $n$; however, in certain conditions, as in Fig. 4(a), we arrive at $\beta_{i}<0$, which may be interpreted as an increase in confinement along the propagation of the mode due to higher loss in the periphery of the core, resulting in a reverse of confinement loss. Note that in obtaining $n$ we have used the transverse $(x, y)$ dependency of $\Omega_{c}$, such that the core is much more transparent in the center and less transparent in the periphery. The other cases that are shown in Fig. 4 correspond to loss. The $\beta_{i}$ for fiber 1 , which is not shown in the figures, was positive and three orders of magnitude larger than those of fiber 2 (Fig. 4). One of the probe modes is represented in Fig. 5.

Based on the results in the previous sections, we search for two distinct cases: one for which the right-hand side of Eq. (12) is very small and we have bright solitons only slightly perturbed by the nonzero imaginary parts of the susceptibility and nonlinear parameter and one for which the right-hand side of Eq. (12) is nonnegligible and we

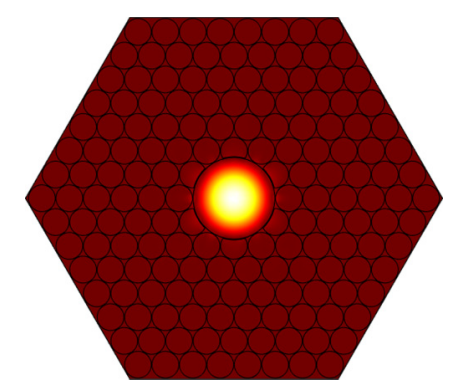

FIG. 5. (Color online) Transverse distribution of the fundamental mode for the probe field at fiber 2 , with $\Delta=0$ and $\Omega_{c}^{p}=7 \times$ $10^{11} \mathrm{~s}^{-1}$. 


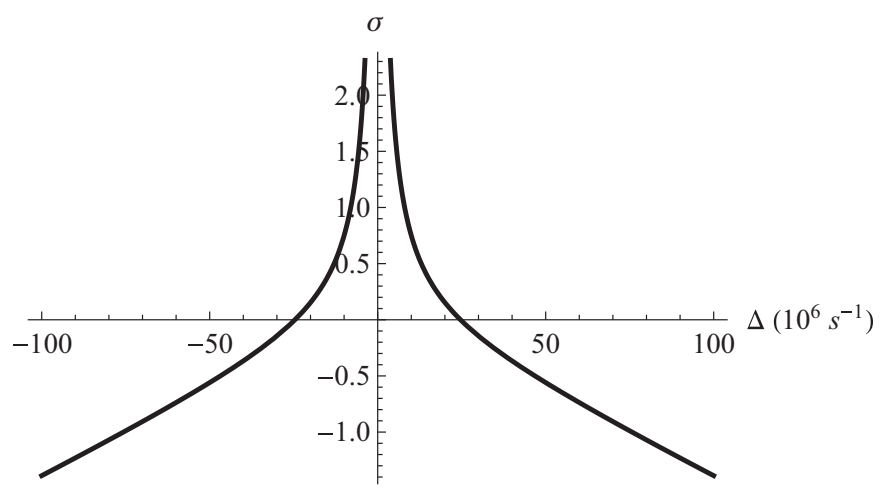

(a)

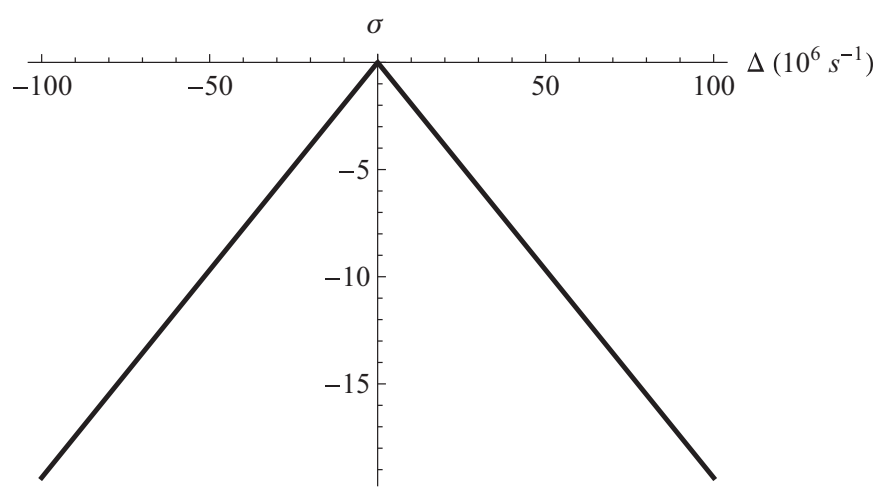

(b)

FIG. 6. Dependence of $\sigma$ with $\Delta$ as calculated using $\sigma=-\operatorname{Im}\left[G\left(\Omega_{c}^{\text {eff }}\right)\right] /\left|\operatorname{Re}\left[G\left(\Omega_{c}^{\text {eff }}\right)\right]\right|$ for (a) case 1 using $\Omega_{c}^{\text {eff }}=7 \times 10^{11} s^{-1}$ and (b) case 2 using $\Omega_{c}^{\text {eff }}=7 \times 10^{10} \mathrm{~s}^{-1}$.

have the conditions of stable pulse solutions. To obtain $\sigma$ we need the imaginary and real parts of $g$ as given by Eq. (11). Since the magnitude of $\beta_{i}$ is considerably lower than the magnitude of $\beta_{r}$, we have $\beta_{p} \approx \beta_{r}$, and $g$ may be written as $g \approx \overline{\mathcal{G}} \int|F|^{4} d x d y /\left(2 \beta_{r} \int|F|^{2} d x d y\right)$, where $\overline{\mathcal{G}}=\int \mathcal{G}|F|^{4} d x d y / \int|F|^{4} d x d y$. Using this approximation, $\sigma=-\operatorname{Im}\left[G\left(\Omega_{c}^{\text {eff }}\right)\right] /\left|\operatorname{Re}\left[G\left(\Omega_{c}^{\text {eff }}\right)\right]\right|$, with $\Omega_{c}^{\text {eff }}$ being an effective value of $\Omega_{c}$ that gives $\overline{\mathcal{G}}$. This expression is independent of the fiber, and its $\Delta$ dependence is shown in Fig. 6 for the two cases considered. For the considered $\Delta$ range, the dependence of $\sigma$ on $\Delta$ is negligibly affected by $\Omega_{c}^{\text {eff }}$ with respect to the global aspect and also to numerical values. If we want $\sigma$ to be small $(|\sigma|<1)$, the detuning $\Delta$ should have a value around $\Delta=$ $\pm 25 \times 10^{6} \mathrm{~s}^{-1}$ in case 1 and around the resonance $(\Delta=0)$ with $|\Delta|$ up to $0.6 \times 10^{6} \mathrm{~s}^{-1}$ in case 2 . We further analyzed the magnitude of the other parameters for case 1 since this case is closer to actual experimental conditions. If we consider fiber $1, v$ cannot be made small around $\pm 25 \times 10^{6} \mathrm{~s}^{-1}$. However, for fiber 2 and for the peak control Rabi frequencies that have been considered, $v$ is small at these $\Delta$ 's, and for $\Delta$ around $25 \times 10^{6} \mathrm{~s}^{-1}, s=1$ and $D=-1$, which enables the possibility of nonlinear Schrödinger solitons. On the other

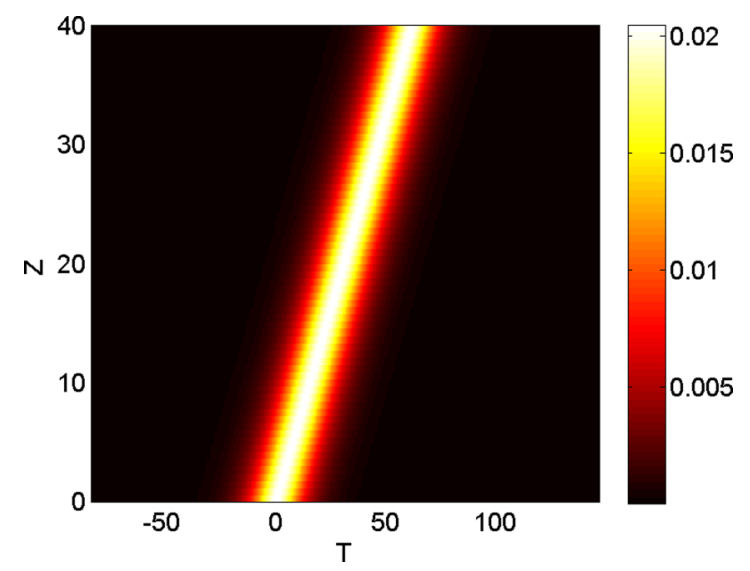

FIG. 7. (Color online) Soliton evolution as simulated using Eq. (12) with $D=1, s=1, \alpha=0.0246, \xi=-0.0011, v=3.5 \times$ $10^{-4}$, and $\sigma=-91.5$ up to $Z=40$. hand, for stable dissipative solitons we need $\alpha+\frac{\xi^{2}}{4 v}>0$. Among the cases that have been considered, the parameters for fiber 2 in the case of high $\gamma_{i j}$ (case 1 ) obey $\alpha+\frac{\xi^{2}}{4 v}>0$ for the major part of the analyzed $\Delta$ range. However, $v>0$ only for $\Delta>6 \times 10^{9} \mathrm{~s}^{-1}$ and $\Delta>9 \times 10^{9} \mathrm{~s}^{-1}$ for $\Omega_{c}^{p}=7 \times 10^{11}$ $\mathrm{s}^{-1}$ and $\Omega_{c}^{p}=1 \times 10^{12} \mathrm{~s}^{-1}$, respectively. Let us consider $\Delta=6.2 \times 10^{9} \mathrm{~s}^{-1}$ for fiber 2 , case 1 , and $\Omega_{c}^{p}=7 \times 10^{11} \mathrm{~s}^{-1}$, which gives $s=1, D=1, \sigma=-90.7$ as calculated using (11) and $v=3.5 \times 10^{-4}$. If we further consider $\tau_{0}=50 \mathrm{ps}$, we get $\alpha=0.0246$ and $\xi=-0.0011$. For this set of parameters, the pulse solution derived above has an amplitude $A=0.02$ and chirp parameter $d=-1.43$. The steady propagation is shown in Fig. 7 up to $Z=40$ for an input consisting of the unperturbed soliton. In this case, the background instability was only significant around $Z=400$, which corresponds to $z=1 \mathrm{~m}$. Note that this dissipative soliton should have a peak power of $3 \mu \mathrm{W}$ since $\gamma_{r}=5.4 \times 10^{4} \mathrm{~W}^{-1} \mathrm{~m}^{-1}$ and will propagate at $1.8 \%$ of the vacuum light velocity.

\section{v. CONCLUSIONS}

We deduced a propagation equation for the optical field on a resonant three-level $\Lambda$ atomic system enclosed in a HC-PCF using a multiscale perturbation approach. The propagation equation is identical to the one deduced elsewhere, but its parameters are dependent on the fiber-guiding effect through the complex propagation constant and transversemode distribution. In certain parameter regions, this equation supports stable dissipative solitons whose analytical form we have deduced. We have also confirmed that for a typical gas and HC-PCF the parameters are such that stable pulses do exist. The stability of these pulses was considered by direct integration of the evolution equation, whose results indicate that even though they are stable in a parameter region that coincides with the region with an unstable background, they propagate steadily for considerable distances. Nevertheless, a careful stability analysis of these solitons will be addressed in a future work, along with a more extensive study of the contribution of the fiber geometry to the propagation characteristics. 


\section{ACKNOWLEDGMENTS}

We acknowledge FCT (Fundação para a Ciência e Tecnologia) for supporting through the Projects No. PTDC/FIS/112624/2009, No. PEst-C/CTM/LA0025/2011, and No. FCOM-01-0124-FEDER-037281.

\section{APPENDIX}

Considering that spontaneous emission occurs only from level 3 to levels 2 and 1, described by $S_{32}$ and $S_{31}$, respectively, and that the energy-conserving dephasing effects are described by $D_{i j}$, the Lindblad term $\hat{\mathcal{L}}\left(\hat{\rho}_{I}\right)$ in $(1)$ is

$$
\left[\begin{array}{c}
S_{31} \rho_{33} \\
-D_{12} \rho_{12} \\
-\frac{S_{31}+S_{32}}{2} \rho_{13}-D_{13} \rho_{13} \\
-D_{12} \rho_{21} \\
S_{32} \rho_{22} \\
-\frac{S_{31}+S_{32}}{2} \rho_{23}-D_{23} \rho_{23} \\
-\frac{S_{31}+S_{32}}{2} \rho_{31}-D_{13} \rho_{31} \\
-\frac{S_{31}+S_{32}}{2} \rho_{32}-D_{23} \rho_{32} \\
-\left(S_{31}+S_{32}\right) \rho_{33}
\end{array}\right] .
$$

The parameter $B$ in Eq. (7) is given by

$$
B=\frac{2 i\left[4\left(\Delta-i \gamma_{12}\right)^{2}+\left|\Omega_{c}\right|^{2}\right]}{\left[4\left(\Delta-i \gamma_{12}\right)\left(\delta_{p}+i \gamma_{13}\right)+\left|\Omega_{c}\right|^{2}\right]^{2}}=i \frac{d L}{d \omega_{p}},
$$

and $G$ and $C$ in Eq. (9) are as follows:

$$
\begin{gathered}
G=\frac{c_{1}+c_{2}\left|\Omega_{c}\right|^{2}+c_{3}\left|\Omega_{c}\right|^{4}}{\gamma_{23} \gamma_{33}\left[-4\left(\Delta+i \gamma_{12}\right)\left(\delta_{p}-i \gamma_{13}\right)+\left|\Omega_{c}\right|^{2}\right]\left[4\left(\Delta-i \gamma_{12}\right)\left(\delta_{p}+i \gamma_{13}\right) \Omega_{c}+\Omega_{c}^{3}\right]^{2}}, \\
c_{1}=-32\left(\Delta-i \gamma_{12}\right)^{2}\left(\Delta+i \gamma_{12}\right) \gamma_{13}\left(\delta_{c}^{2}+\gamma_{23}^{2}\right) \gamma_{33}, \\
c_{2}=-8\left(\Delta-i \gamma_{12}\right)\left[12\left(\Delta^{2}+\gamma_{12}^{2}\right) \gamma_{13} \gamma_{23}-i\left\{\gamma_{12}\left(2 \delta_{c} \gamma_{13}-\delta_{p} \gamma_{23}+i \gamma_{13} \gamma_{23}\right)+\Delta\left[\delta_{c}\left(\delta_{p}+\delta_{c}-i \gamma_{13}\right)+2 \gamma_{13} \gamma_{23}+\gamma_{23}^{2}\right]\right\} \gamma_{33}\right], \\
c_{3}=2 i\left\{12 \Delta^{2} \gamma_{23}-\gamma_{12}\left(\gamma_{13}+\gamma_{23}\right) \gamma_{33}+\Delta\left[-\left(\delta_{p}+\delta_{c}\right) \gamma_{33}-2 i \gamma_{23}\left(6 \gamma_{12}+\gamma_{33}\right)\right]\right\}, \\
C=\frac{8\left\{4\left(\Delta-i \gamma_{12}\right)^{3}+\left[2 \delta_{c}-3 \delta_{p}-i\left(2 \gamma_{12}+\gamma_{13}\right)\right]\left|\Omega_{c}\right|^{2}\right\}}{\left[4\left(\Delta-i \gamma_{12}\right)\left(\delta_{p}+i \gamma_{13}\right)+\left|\Omega_{c}\right|^{2}\right]^{3}}=-\frac{1}{2} \frac{d^{2} L}{d \omega_{p}^{2}} .
\end{gathered}
$$

[1] S. E. Harris, Phys. Today 50(7), 36 (1997).

[2] S. E. Harris, J. E. Field, and A. Imamoglu, Phys. Rev. Lett. 64, 1107 (1990).

[3] K. J. Boller, A. Imamoglu, and S. E. Harris, Phys. Rev. Lett. 66, 2593 (1991).

[4] L. V. Hau, S. E. Harris, Z. Dutton, and C. H. Behroozi, Nature (London) 397, 594 (1999).

[5] A. I. Lvovsky, B. C. Sanders, and W. Tittel, Nat. Photonics 3, 706 (2009).

[6] S. E. Harris and Y. Yamamoto, Phys. Rev. Lett. 81, 3611 (1998).

[7] M. Bajcsy, S. Hofferberth, V. Balic, T. Peyronel, M. Hafezi, A. S. Zibrov, V. Vuletic, and M. D. Lukin, Phys. Rev. Lett. 102, 203902 (2009).
[8] C. Liu, Z. Dutton, C. H. Behroozi, and L. V. Hau, Nature (London) 409, 490 (2001).

[9] D. F. Phillips, A. Fleischhauer, A. Mair, R. L. Walsworth, and M. D. Lukin, Phys. Rev. Lett. 86, 783 (2001).

[10] J. Xu and G. Huang, Opt. Express 21, 5149 (2013).

[11] C. Hang and V. V. Konotop, Phys. Rev. A 81, 053849 (2010).

[12] S. Ghosh, J. E. Sharping, D. G. Ouzounov, and A. L. Gaeta, Phys. Rev. Lett. 94, 093902 (2005).

[13] F. Benabid, P. Light, F. Couny, and P. Russell, Opt. Express 13, 5694 (2005).

[14] S. Ghosh, A. R. Bhagwat, C. K. Renshaw, S. Goh, A. L. Gaeta, and B. J. Kirby, Phys. Rev. Lett. 97, 023603 (2006). 
[15] G. Huang, L. Deng, and M. G. Payne, Phys. Rev. E 72, 016617 (2005).

[16] J. M. Soto-Crespo, N. N. Akhmediev, V. V. Afanasjev, and S. Wabnitz, Phys. Rev. E 55, 4783 (1997).

[17] N. R. Pereira and L. Stenflo, Phys. Fluids 20, 1733 (1977).

[18] N. N. Akhmediev, V. V. Afanasjev, and J. M. Soto-Crespo, Phys. Rev. E 53, 1190 (1996).
[19] J. Borhanian, Plasma Phys. Controlled Fusion 55, 105012 (2013).

[20] D. J. Kaup and A. C. Newell, Proc. R. Soc. London, Ser. A 361, 413 (1978).

[21] V. I. Karpman, Phys. Scr. 20, 462 (1979).

[22] D. A. Steck (unpublished), http://steck.us/alkalidata

[23] COMSOL MULTIPHYSICS, version 4.4, Comsol AB, Stockholm, 2013. 